\title{
Diffusion-Controlled Solid-State Formation of CoSb Phase from Co/Sb-Multilayered Nanowires
}

\author{
Seong Gi Jeon, ${ }^{1}$ Ho Sun Shin, ${ }^{2}$ Jin Yu, ${ }^{1}$ and Jae Yong Song ${ }^{2,3}$ \\ ${ }^{1}$ Department of Materials Science and Engineering, Korea Advanced Institute of Science and Technology, \\ Daejeon 305-701, Republic of Korea \\ ${ }^{2}$ Center for Nanocharacterization, Korea Research Institute of Standards and Science, \\ Daejeon 305-340, Republic of Korea \\ ${ }^{3}$ Department of Nano Science, University of Science and Technology, Daejeon 305-350, Republic of Korea
}

Correspondence should be addressed to Jae Yong Song, jysong@kriss.re.kr

Received 12 June 2012; Accepted 31 July 2012

Academic Editor: Yanqiu Zhu

Copyright (c) 2012 Seong Gi Jeon et al. This is an open access article distributed under the Creative Commons Attribution License, which permits unrestricted use, distribution, and reproduction in any medium, provided the original work is properly cited.

The physical properties of materials on a nanometer scale are known to be different from those of bulk form due to dimensional confinement effects and high specific surface-to-volume ratio. In this study, the size effects on the diffusion and reaction at the interface of dissimilar nanowires (NWs) were investigated. Co/Sb-multilayered NWs of $15 \pm 1$ and $19 \pm 2$ nm in radius have been grown within anodic aluminum oxide templates using a pulsed potentiodynamic electrodeposition method. XRD and TEM results demonstrated that the multilayered NWs were transformed to a CoSb phase through a solid-state reaction at the temperature in the range of 653 to $693 \mathrm{~K}$. The kinetics of the solid-state reaction was analyzed and found to be diffusion controlled at the interface between $\mathrm{Co}$ and $\mathrm{Sb}$ phases. The reaction was controlled by the dominant diffusion of Sb atoms. The activation energies for the solid-state reaction were estimated to be 0.9 and $0.7 \mathrm{eV} /$ atom for the multilayered NWs with radii of 15 and $19 \mathrm{~nm}$, respectively.

\section{Introduction}

Synthesis of one-dimensional nanomaterials is a rapidly growing research area with applications in energy generating, energy storage, and environmental sensing [1]. It is wellknown that materials on a nanometer scale have exceptional physical/chemical properties in comparison with the bulk form. To date, a number of studies have examined the size effects on surface energies [2], elastic moduli [3, 4], melting temperatures [5], superconductivity [6], and lattice deformation $[7,8]$ for nanowires (NWs). The size-dependent properties are due to the dimensional confinement, high surface-to-volume ratio, and surface relaxation related to imperfection of coordination number on the surface [9].

In a similar vein, the metallurgical phenomena of phase transformation, solid-state diffusion, and melting behavior can be affected by the size effects because the high surfaceto-volume ratio can enhance the atomic mobility at the surface with certain imperfect coordination number $[10,11]$. Recently, it has been reported that phase transformation and solid-state diffusion on a nanometer scale are greatly influenced by confined-nanoscale geometry [12-14].

Template-based electrochemical synthesis of NWs is generally used because this process has several merits of controlling the diameter of NWs, low cost, and large-area deposition. Recently, pulsed electrochemical deposition has been used to control the composition of alloy deposits within templates [15-18].

We selected $\mathrm{Co}$ and $\mathrm{Sb}$ in order to investigate the solidstate reaction in the form of NWs because the reaction between $\mathrm{Co}$ and $\mathrm{Sb}$ can result in the formation of several stoichiometric compounds such as $\mathrm{CoSb}, \mathrm{CoSb}_{2}$, and $\mathrm{CoSb}_{3}$; these compounds have attracted much attention in the fields of secondary Li-ion batteries and thermoelectric devices $[19,20]$, because a vertical configuration of one-dimensional nanostructure offers distinct benefits over a lateral configuration in its utilization of a high specific surface area. As an anode of secondary Li-ion batteries, the small radius of NWs allows for better accommodation of large-volume changes without the initiation of fracture. And the high 


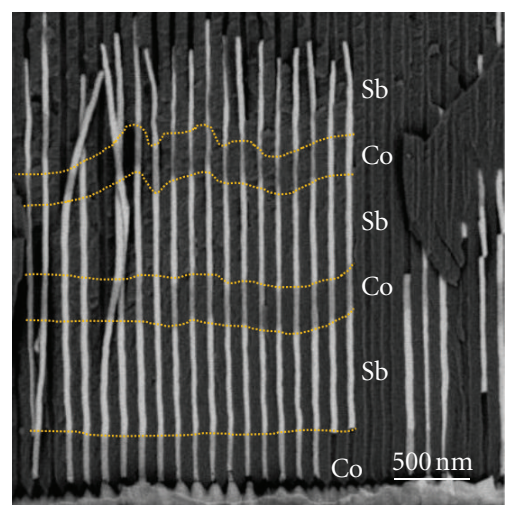

(a)

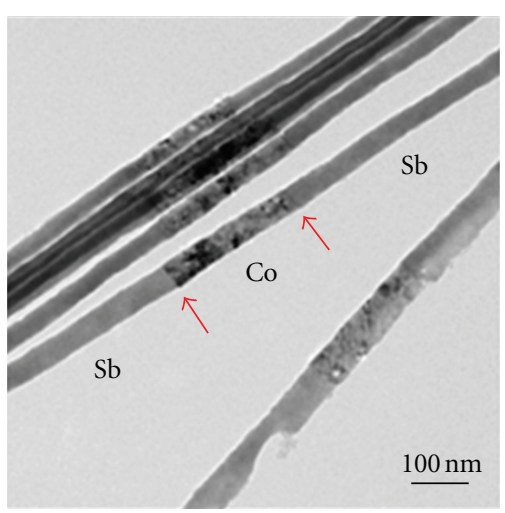

(b)

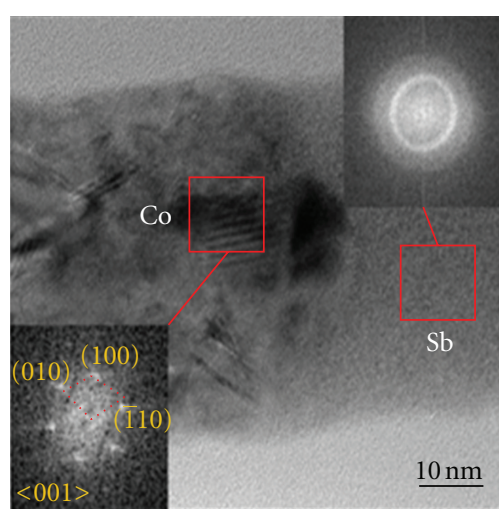

(c)

Figure 1: (a) SEM image, (b) bright field TEM image, and (c) HR-TEM image at the interface between Co and Sb segments of Co/Sbmultilayered nanowires $\left(r_{\mathrm{NW}}=15 \mathrm{~nm}\right)$. The insets denote the FFT images of the marked areas.

specific surface area of NWs can contribute to the lowering of the lattice thermal conductivity and the enhancement of thermoelectric figure of merit [19].

In this study, we investigated an alternative growth method of a Co and Sb NW array using pulsed potentiodynamic electrodeposition based on the different reduction potentials of $\mathrm{Co}$ and $\mathrm{Sb}$ cations. The diffusion-controlled solid-state reaction, confined in a cross-sectional area of several tens of nanometers, was analyzed.

\section{Experimental Procedure}

Anodic aluminum oxide (AAO) templates were synthesized on $\mathrm{Al}$ foil $(99.99 \%)$ by a two-step anodization process in $0.3 \mathrm{M}$ oxalic acid $(40 \mathrm{~V})$ at $274 \mathrm{~K}$. Then, the remaining $\mathrm{Al}$ foil was removed in an etching solution composed of $0.1 \mathrm{M} \mathrm{CuCl}_{2}$ and $20 \mathrm{wt} \% \mathrm{HCl}$. The barrier layer of the $\mathrm{AAO}$ template was removed in $0.5 \mathrm{M} \mathrm{H}_{3} \mathrm{PO}_{4}$ solution at $303 \mathrm{~K}$ for the following electrodeposition process. A $150 \mathrm{~nm}$ thick $\mathrm{Au}$ layer was deposited on one side of the AAO template using an RF sputtering system; this layer served as a working electrode in the electrochemical deposition process. Using a pore-widening process [5], we fabricated two AAO templates with a pore radius of $15 \pm 1$ and $19 \pm 2 \mathrm{~nm}$. The detailed procedures of preparing the AAO templates can be found in previous reports $[5,8]$.

Electrodeposition was carried out using a potentio/galvanostat (Solartron 1280z) as a power source with a three-electrode setup; a saturated $\mathrm{Ag} / \mathrm{AgCl}$ electrode as a reference; a Pt wire as a counter electrode; an AAO as a working electrode. The electrolyte $(\mathrm{pH} 1.5)$ for the electrodeposition of $\mathrm{Co} / \mathrm{Sb}$-multilayered NWs was composed of $0.1 \mathrm{M} \mathrm{CoSO}_{4} \cdot 7 \mathrm{H}_{2} \mathrm{O}, 0.01 \mathrm{M} \mathrm{SbCl}_{3}$, and $0.14 \mathrm{M}$ tartaric acid. We consecutively conducted alternative electrodepositions of $\mathrm{Co}$ and $\mathrm{Sb}$ for the multilayered $\mathrm{Co} / \mathrm{Sb} \mathrm{NWs}$ in the pulsed potentiodynamic mode. We used reduction potentials $\left(V_{R}\right)$ of -1.3 and $-0.8 \mathrm{~V}$ (versus saturated $\mathrm{Ag} / \mathrm{AgCl}$ ) for the growth of $\mathrm{Co}$ and $\mathrm{Sb}$ segments, respectively. When changing the $V_{R}$ to -1.3 and $-0.8 \mathrm{~V}$, Co and $\mathrm{Sb}$ phases, respectively, were deposited onto the AAO templates. From
SEM observation, the respective growth rates of Co and $\mathrm{Sb}$ NWs were experimentally determined to be 10 and $2 \mathrm{~nm} \cdot \mathrm{s}^{-1}$.

The NWs in the AAO templates were heat treated in a vacuum of $4 \times 10^{-6}$ Torr in a temperature range of 653 to $693 \mathrm{~K}$. A field-emission scanning electron microscope (SEM, Hitachi S4800), X-ray diffractometer (XRD, Rigaku, D/MAX-RC, $12 \mathrm{~kW}$ ), and transmission electron microscope (TEM, Tecnai G2 F30, $300 \mathrm{kV}$ ) were used to analyze the crystal structures and morphologies of the multilayered NWs before and after the heat treatment. For the purpose of TEM characterization, the AAO structure was dissolved in a $1 \mathrm{M}$ $\mathrm{NaOH}$ solution. The remaining NWs were rinsed in distilled water and were dispersed in ethanol. We prepared the TEM specimen by dropping the NW dispersed ethanol solution on a TEM grid.

\section{Results and Discussion}

Figure 1(a) shows a typical cross-sectional SEM image of the $\mathrm{Co} / \mathrm{Sb}$-multilayered NWs with a radius of $r_{\mathrm{NW}}=15 \mathrm{~nm}$ within the AAO template. At first, the Co segment (dark region) was deposited at the bottom of the template, and then the $\mathrm{Sb}$ segment (bright region) was consecutively deposited at the top of the Co segment along the pore channels of the template. After three cycles of $V_{R}$ modulation from $-1.3 \mathrm{~V}$ to $-0.8 \mathrm{~V}$, three bilayers of $\mathrm{Co} / \mathrm{Sb}$ were repeatedly synthesized, as shown in Figure 1(a). The interface boundaries are indicated by the dotted yellow lines shown in Figure 1(a). The lengths of the Co and Sb segments were $300 \pm 40$ and $600 \pm 50 \mathrm{~nm}$, respectively. In Figure 1(b), a bright-field TEM image shows that each segment of $\mathrm{Co}$ and $\mathrm{Sb}$ was densely interconnected at the interfaces. Figure 1(c) shows a high-resolution (HR) TEM image of the interface between $\mathrm{Co}$ and $\mathrm{Sb}$ segments. The insets indicate the fast Fourier transformation (FFT) images corresponding to the marked areas in the Co and Sb segments, respectively. The FFT image from the $\mathrm{Sb}$ segment shows a diffuse ring pattern indicating that the $\mathrm{Sb}$ segment is in an amorphous state. The FFT image from the Co segment shows that the Co segment 


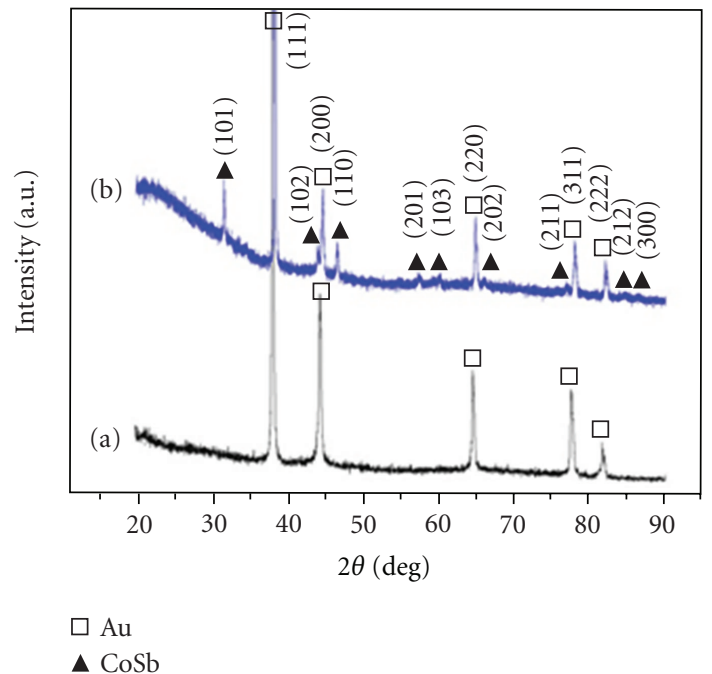

FIGURE 2: XRD patterns of Co/Sb-multilayered nanowires $\left(r_{\mathrm{NW}}=\right.$ $15 \mathrm{~nm}$ ) (a) in the as-prepared state and (b) after heat treatment at $673 \mathrm{~K}$ for $10 \mathrm{~min}$

has a hexagonal closed packed crystal structure with a [001]zone axis. Although the marked area of the Co segment was analyzed and found to be of crystalline phase, it should be mentioned that most of the other parts of the Co segment have an amorphous structure. From the HR TEM analysis shown in Figure 1(c), it can be supposed that the Co segment has a mixed phase of Co nanocrystals (grain size less than $10 \mathrm{~nm}$ ), in a very limited region, and Co in an amorphous phase. The TEM results were in a good agreement with the XRD results shown in Figure 2(a). Figure 2(a) shows the XRD pattern of the Co/Sb-multilayered NWs $\left(r_{\mathrm{NW}}=\right.$ $15 \mathrm{~nm}$ ) in the as-prepared state. In Figure 2(a), there are no reflection peaks from elemental $\mathrm{Co}$ and $\mathrm{Sb}$, or $\mathrm{Co}-\mathrm{Sb}$ compound phases, indicating that $\mathrm{Co}$ and $\mathrm{Sb}$ segments in the as-prepared state were in an amorphous state. Figure 2(b) shows the XRD pattern of the Co/Sb-multilayered NWs of $r_{\mathrm{NW}}=15 \mathrm{~nm}$ after the heat treatment at $673 \mathrm{~K}$ for $10 \mathrm{~min}$. The XRD pattern of the Co/Sb-multilayered NWs of $r_{\mathrm{NW}}=19 \mathrm{~nm}$ after heat treatment is not shown here because it was almost the same as that of the NWs of $r_{\mathrm{NW}}=$ $15 \mathrm{~nm}$. During the heat treatment, the transformation of the $\mathrm{Co} / \mathrm{Sb}$-multilayered structure into $\mathrm{CoSb}$ phase occurred at the interface between $\mathrm{Co}$ and $\mathrm{Sb}$, regardless of the wire radius, as shown in Figure 2(b).

Figure 3 displays the bright-field and HR TEM images of $\mathrm{Co} / \mathrm{Sb}$-multilayered NWs $\left(r_{\mathrm{NW}}=15 \mathrm{~nm}\right)$ after heat treatment at $673 \mathrm{~K}$. It was observed that the Co segment was shortened from the initial length $(300 \mathrm{~nm})$ to $130 \mathrm{~nm}$, and that the CoSb segment (about $230 \mathrm{~nm}$ in length) formed after the heat treatment, as can be seen in Figure 3(a). The HR TEM image and the corresponding FFT result shown in the inset of Figure 3(b) show a crystalline segment indexed as a hexagonal $\mathrm{CoSb}$ phase resulting from the solid-state reaction between $\mathrm{Co}$ and $\mathrm{Sb}$ segments. The CoSb segment was disconnected from the Sb segment, as shown in Figures 4 and 5 , while the interface between Co and CoSb segments was

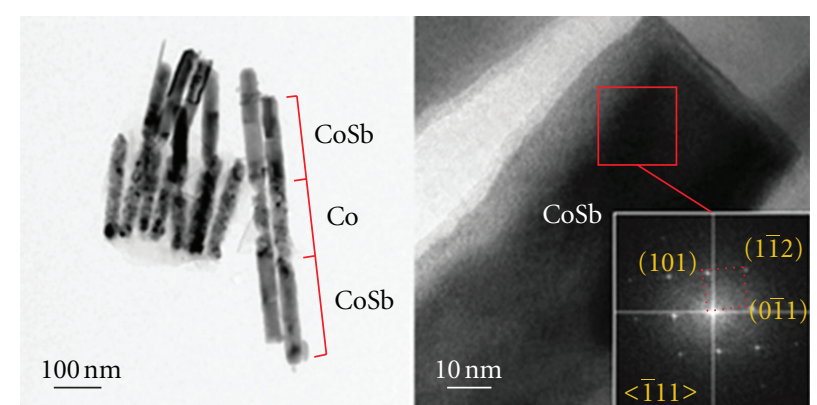

FIgURE 3: (a) Bright field TEM image and (b) HR-TEM image of $\mathrm{Co} / \mathrm{Sb}$-multilayered nanowires $\left(r_{\mathrm{NW}}=15 \mathrm{~nm}\right)$ after heat treatment at $673 \mathrm{~K}$ for $10 \mathrm{~min}$. The inset denotes the FFT image of the marked area of the CoSb segment.

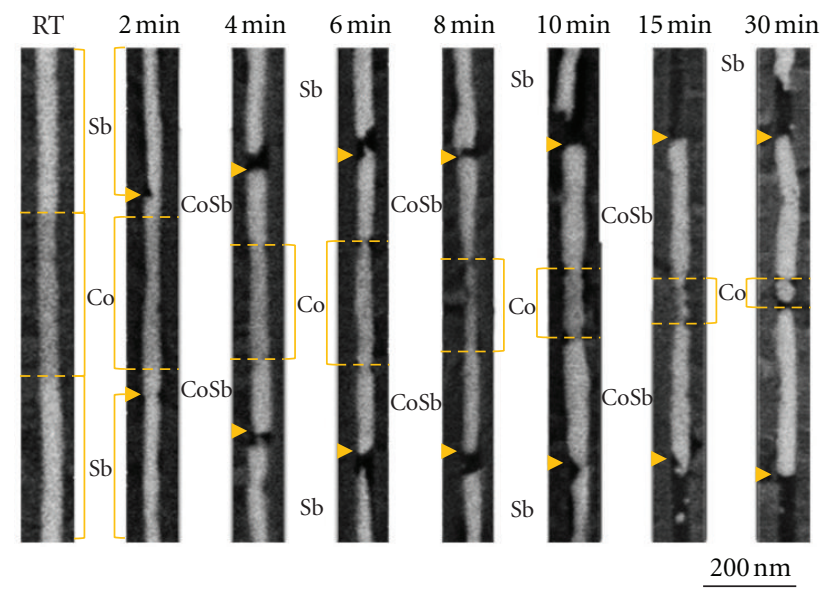

FIGURE 4: SEM images of the multilayered nanowires $\left(r_{\mathrm{NW}}=\right.$ $15 \mathrm{~nm}$ ) with isothermal heat treatment time of up to $30 \mathrm{~min}$ at $673 \mathrm{~K}$.

connected despite the formation of the small notches that are shown in Figures 3 to 5. This suggests that the diffusion of $\mathrm{Sb}$ atoms toward the Co segment was dominant during the solid-state reaction. Although the dominant diffusion of $\mathrm{Sb}$ atoms causes a molar volume expansion, it is presumed that the volume expansion occurs along the longitudinal direction of the pores due to the confinement within the nanotemplates. In Figures 4 and 5 , it should be noted that the interface between $\mathrm{Sb}$ and CoSb segments started to disconnect after heat treatment for $4 \mathrm{~min}$. Despite this disconnection, the CoSb segments grew with heat treatment time up to $30 \mathrm{~min}$. The $\mathrm{Sb}$ atoms needed for the formation of the CoSb segment are presumed to be supplied by the sublimation of the Sb segment as well as by surface diffusion along the wall surface of the AAO template. Previously, it was reported that $\mathrm{Sb}$ can be sublimated in vacuum at $650 \mathrm{~K}$ [21] and that $\mathrm{Sb}$ NWs sublimated at $769 \mathrm{~K}$ in ambient atmosphere [22]. Accordingly, the heat treatment was conducted in the range of 653 to $693 \mathrm{~K}$ for proper measurement of the solidstate reaction rate.

The transformation kinetics from $\mathrm{Co} / \mathrm{Sb}$-multilayer to $\mathrm{CoSb}$ phase was investigated by the isothermal heat treatment of the Co/Sb-multilayered NWs. As can be seen in 


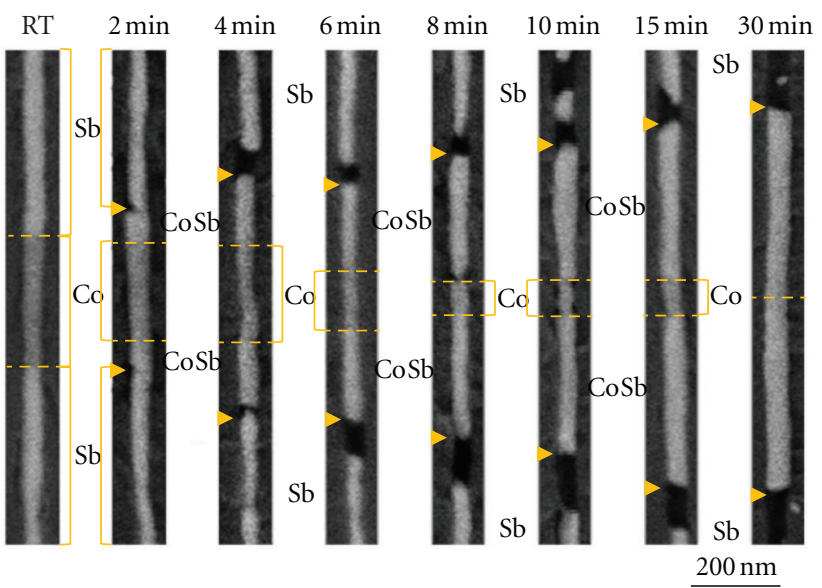

FIGURE 5: SEM images of the multilayered nanowires $\left(r_{\mathrm{NW}}=\right.$ $19 \mathrm{~nm}$ ) with isothermal heat treatment time of up to $30 \mathrm{~min}$ at $673 \mathrm{~K}$.

Figure 4, the Co segment was transformed to a CoSb segment by the dominant diffusion of $\mathrm{Sb}$ atoms in the $\mathrm{Co} / \mathrm{Sb}$ multilayered NWs with $r_{\mathrm{NW}}$ of $15 \mathrm{~nm}$. The length of the Co segment (dark region between the dotted lines) decreased with the time, while that of the CoSb segment (between the dotted lines and to the triangle) increased with the heat treatment time out of the Co segment ends. After heat treatment for $30 \mathrm{~min}$, a $40 \mathrm{~nm}$ length Co segment remained, while a $270 \mathrm{~nm}$ length $\mathrm{CoSb}$ segment formed, as shown in Figure 4. In the case of Co/Sb-multilayered NWs of $r_{\mathrm{NW}}=$ $19 \mathrm{~nm}$, very similar reactions were observed, as can be seen in Figure 5. Interestingly, the Co segment was almost consumed in forming the CoSb segment after $30 \mathrm{~min}$, as can be seen in Figure 5. After heat treatment, the lengths of the CoSb NWs were observed to be about $350 \mathrm{~nm}$.

As can be seen in Figures 4 and 5, the lengths $(\lambda)$ of the CoSb segments were measured with the variation of the heat treatment time. The length of the CoSb segment was determined as the average value out of ten measurements of the length of each NW. Figure 6 shows the variations of $\mathrm{CoSb}$ length with the heat treatment time. The variations of CoSb lengths with the time showed similar behavior for the two NWs with radii of $r_{\mathrm{NW}}=15$ and $19 \mathrm{~nm}$. The variations of CoSb lengths with the time were fitted by the following relation:

$$
\lambda=(D t)^{n} .
$$

Here, $D$ and $t$ denote a diffusion coefficient and diffusion time; $n$ is a diffusion exponent. When $n=0.5$, the variation of the CoSb lengths was in good agreement with the fitting line. It is wellknown that $n=1$ implies a reactioncontrolled process, while $n=0.5$ indicates a diffusioncontrolled process [23]. Therefore, the data and fitting shown in Figure 6 suggest that the solid-state reaction of the $\mathrm{CoSb}$ formation occurred in a diffusion-controlled mode. The overall reaction rate was controlled by Sb diffusion through the CoSb phase, which was evidenced by the formation of

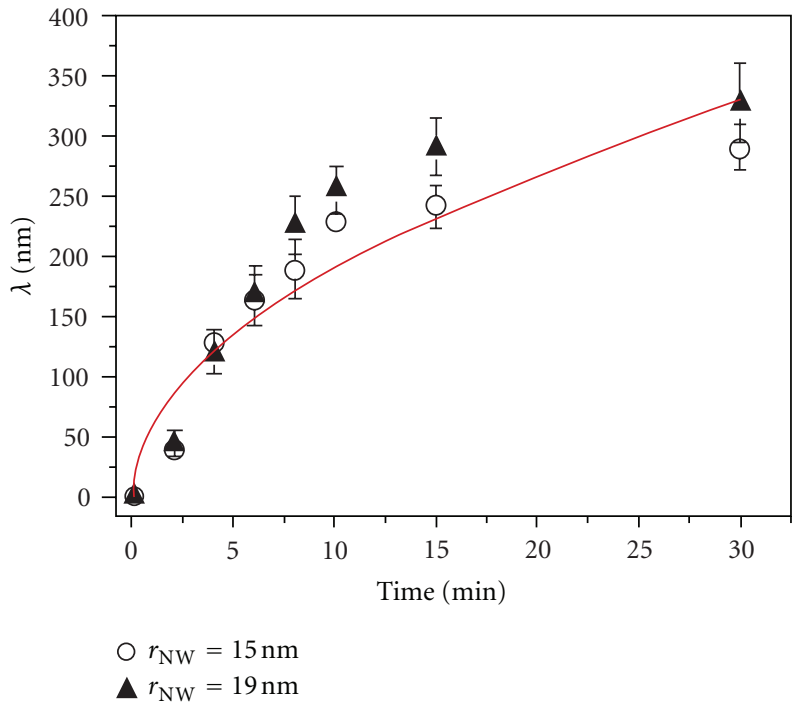

FIgURE 6: Variations of CoSb length $(\lambda)$ with isothermal heat treatment time at $673 \mathrm{~K}$. The curve (red line) was fitted according to (1) and $n=0.5$.

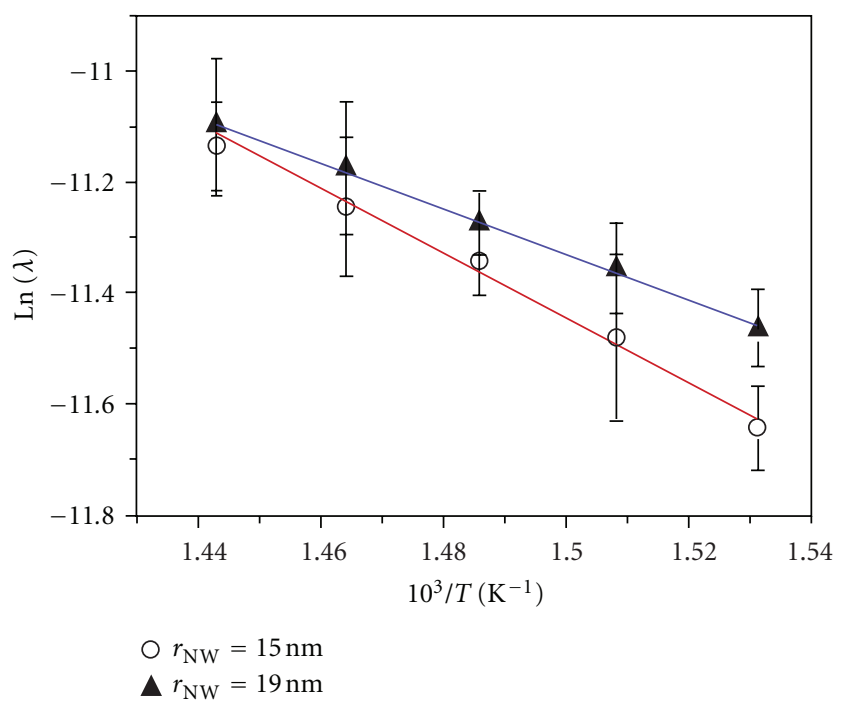

Figure 7: Variations of CoSb length on log scale (in unit of $\mathrm{cm}$ ) with inverse heat treatment temperature in the range of 653 to $693 \mathrm{~K}$.

the notches beside the Co segment and the disconnection between the $\mathrm{CoSb}$ and $\mathrm{Sb}$ phases.

Using the Arrhenius relation of $D=D_{0} \exp (-Q / k T),(1)$ is reduced to the following relation:

$$
\ln \lambda=A-\frac{Q}{(2 k T)} .
$$

Here, $A, k, T$, and $Q$ denote a constant, the Boltzmann constant, temperature, and the activation enthalpy for solidstate diffusion, respectively. The lengths of the CoSb segment were measured by varying the heat treatment temperature in the range of 653 to $693 \mathrm{~K}$. The variations of $\ln \lambda$ with the inverse temperature, $1 / T$, were linearly fitted in Figure 7. 
According to (2), the slopes of the linear fits in the plot of $\ln \lambda$ with $1 / T$ are equal to the values of $Q /(2 k)$. Thus, the activation enthalpies were estimated to be 0.9 and $0.7 \mathrm{eV} /$ atom for the Co/Sb-multilayered NWs of $r_{\mathrm{NW}}=15$ and $19 \mathrm{~nm}$, respectively. The estimated activation enthalpies indicate the barrier energy needed for the diffusion through the CoSb phase because the reaction was controlled by the diffusion of $\mathrm{Sb}$ atoms, as discussed above. Considering the overlapped standard deviations of data shown in Figure 7, it is supposed that the activation enthalpies of the solid-state reactions for the two NW radii were not very different from each other. They are lower than the activation enthalpies (2.7 and $1.3 \mathrm{eV} /$ atom) needed for the diffusion of Co in bulk Fe and $\mathrm{Sb}$ in bulk $\mathrm{Sn}$, respectively $[24,25]$. This suggests that the activation energy for atomic diffusion on a nanometer scale can be slightly lower than that in bulk form, which is in agreement with previous reports $[10,11]$.

\section{Conclusion}

We have produced a Co/Sb-multilayered NW array within an AAO template using pulsed potentiodynamic electrodeposition. After heat treatment, the amorphous $\mathrm{Co} / \mathrm{Sb}$ multilayered NWs transformed into CoSb crystalline phase through a solid-state reaction. We analyzed the solid-state reaction, which was confined in the nanotemplate, in order to understand the phase transformation kinetics on a nanometer scale. From the heat treatment experiment, it was confirmed that the solid-state reaction between $\mathrm{Co}$ and $\mathrm{Sb}$ segments is diffusion controlled and that the Sb diffusion through the CoSb phase is the dominant process. The activation enthalpies for the solid-state reaction were estimated to be $0.9 \mathrm{eV} /$ atom for $r_{\mathrm{NW}}=15 \mathrm{~nm}$ and $0.7 \mathrm{eV} /$ atom for $r_{\mathrm{NW}}=19 \mathrm{~nm}$. It is supposed that the activation enthalpies for solid-state diffusion in the NWs are lower than those for the bulk form. Further experimental studies on the effects of wire radius on the solid-state diffusion kinetics are needed to quantitatively analyze the size effects.

\section{Acknowledgment}

This work was supported by Basic Science Research Program through the National Research Foundation of Korea funded by the MEST (Project no. 2012-000181).

\section{References}

[1] Y. Xia, P. Yang, Y. Sun et al., "One-dimensional nanostructures: synthesis, characterization, and applications," Advanced Materials, vol. 15, no. 5, pp. 353-389, 2003.

[2] G. Ouyang, X. Tan, and G. Yang, "Thermodynamic model of the surface energy of nanocrystals," Physical Review B, vol. 74, no. 19, Article ID 195408, 2006.

[3] C. Q. Chen, Y. Shi, Y. S. Zhang, J. Zhu, and Y. J. Yan, "Size dependence of Young's modulus in $\mathrm{ZnO}$ nanowires," Physical Review Letters, vol. 96, no. 7, Article ID 075505, 4 pages, 2006.

[4] E. C. C. M. Silva, L. Tong, S. Yip, and K. J. Van Vliet, "Size effects on the stiffness of silica nanowires," Small, vol. 2, no. 2, pp. 239-243, 2006.
[5] H. S. Shin, J. Yu, and J. Y. Song, "Size-dependent thermal instability and melting behavior of Sn nanowires," Applied Physics Letters, vol. 91, no. 17, Article ID 173106, 2007.

[6] M. Tian, J. Wang, J. S. Kurtz et al., "Dissipation in quasi-onedimensional superconducting single-crystal Sn nanowires," Physical Review B, vol. 71, no. 10, Article ID 104521, 7 pages, 2005.

[7] J. Eymery, V. Favre-Nicolin, L. Fröberg, and L. Samuelson, "Xray measurements of the strain and shape of dielectric/metallic wrap-gated InAs nanowires," Applied Physics Letters, vol. 94, no. 13, Article ID 131911, 2009.

[8] H. S. Shin, J. Yu, J. Y. Song, H. M. Park, and Y. S. Kim, "Origins of size-dependent lattice dilatation in tetragonal Sn nanowires: surface stress and growth stress," Applied Physics Letters, vol. 97, no. 13, Article ID 131903, 2010.

[9] C. Q. Sun, "Size dependence of nanostructures: impact of bond order deficiency," Progress in Solid State Chemistry, vol. 35, no. 1, pp. 1-159, 2007.

[10] A. I. Persson, M. W. Larsson, S. Stenström, B. J. Ohlsson, L. Samuelson, and L. R. Wallenberg, "Solid-phase diffusion mechanism for GaAs nanowire growth," Nature Materials, vol. 3, no. 10, pp. 677-681, 2004.

[11] Y. C. Chou, W. W. Wu, L. J. Chen, and K. N. Tu, "Homogeneous nucleation of epitaxial $\mathrm{CoSi}_{2}$ and NiSi in Si nanowires," Nano Letters, vol. 9, no. 6, pp. 2337-2342, 2009.

[12] Y. C. Chou, W. W. Wu, S. L. Cheng et al., "In-situ TEM Observation of repeating events of nucleation in epitaxial growth of nano $\mathrm{CoSi}_{2}$ in nanowires of Si," Nano Letters, vol. 8, no. 8, pp. 2194-2199, 2008.

[13] V. C. Holmberg, M. G. Panthani, and B. A. Korgel, "Phase transitions, melting dynamics, and solid-state diffusion in a nano test tube," Science, vol. 326, no. 5951, pp. 405-407, 2009.

[14] A. M. Gusak and K. N. Tu, "Interaction between the Kirkendall effect and the inverse Kirkendall effect in nanoscale particles," Acta Materialia, vol. 57, no. 11, pp. 3367-3373, 2009.

[15] X. Dou, Y. Zhu, X. Huang, A. Li, and G. Li, "Effective deposition potential induced size-dependent orientation growth of Bi-Sb alloy nanowire arrays," Journal of Physical Chemistry B, vol. 110, no. 43, pp. 21572-21575, 2006.

[16] W. J. Li, W. L. Yu, and C. Y. Yen, "Pulsed electrodeposition of $\mathrm{Bi}_{2} \mathrm{Te}_{3}$ and $\mathrm{Bi}_{2} \mathrm{Te}_{3} / \mathrm{Te}$ nanowire arrays from a DMSO solution," Electrochimica Acta, vol. 58, no. 1, pp. 510-515, 2011.

[17] L. Chen, H. Hu, Y. Li, G. Chen, S. Yu, and G. Wu, "Ordered $\mathrm{CoSb}_{3}$ nanowire arrays synthesized by electrodeposition," Chemistry Letters, vol. 35, no. 2, pp. 170-171, 2006.

[18] H. Cheng, H. H. Hng, J. Ma, and X. J. Xu, "Effects of various deposition parameters on the co-deposition behavior of cobalt antimony in citric-based solution," Journal of Materials Research, vol. 23, no. 11, pp. 3013-3020, 2008.

[19] M. S. Dresselhaus, G. Chen, M. Y. Tang et al., "New directions for low-dimensional thermoelectric materials," Advanced Materials, vol. 19, no. 8, pp. 1043-1053, 2007.

[20] C. K. Chan, H. Peng, G. Liu et al., "High-performance lithium battery anodes using silicon nanowires," Nature Nanotechnology, vol. 3, no. 1, pp. 31-35, 2008.

[21] B. V. L'vov and A. V. Novichikhin, "Quantitative interpretation of the evaporation coefficients for the decomposition or sublimation of some substances in vacuo," Thermochimica Acta, vol. 290, no. 2, pp. 239-251, 1997.

[22] X. Zhang, Y. Ding, Y. Zhang, Y. Hao, G. Meng, and L. Zhang, "Thermal behavior of antimony nanowire arrays embedded in anodic aluminum oxide template," Journal of Thermal Analysis and Calorimetry, vol. 89, no. 2, pp. 493-497, 2007. 
[23] J. W. Christian, The Theory of Transformation in Metals and Alloys, Pergamon, Oxford, UK, 1975.

[24] F. H. Huang and H. B. Huntington, "Diffusion of $\mathrm{Sb}^{124}, \mathrm{Cd}^{109}$, $\mathrm{Sn}^{113}$, and $\mathrm{Zn}^{65}$ in tin," Physical Review B, vol. 9, no. 4, pp. 1479-1488, 1974.

[25] D. W. James and G. M. Leak, "Self-diffusion and diffusion of cobalt in alpha and delta-iron," Philosophical Magazine, vol. 14, no. 130, pp. 701-713, 1966. 

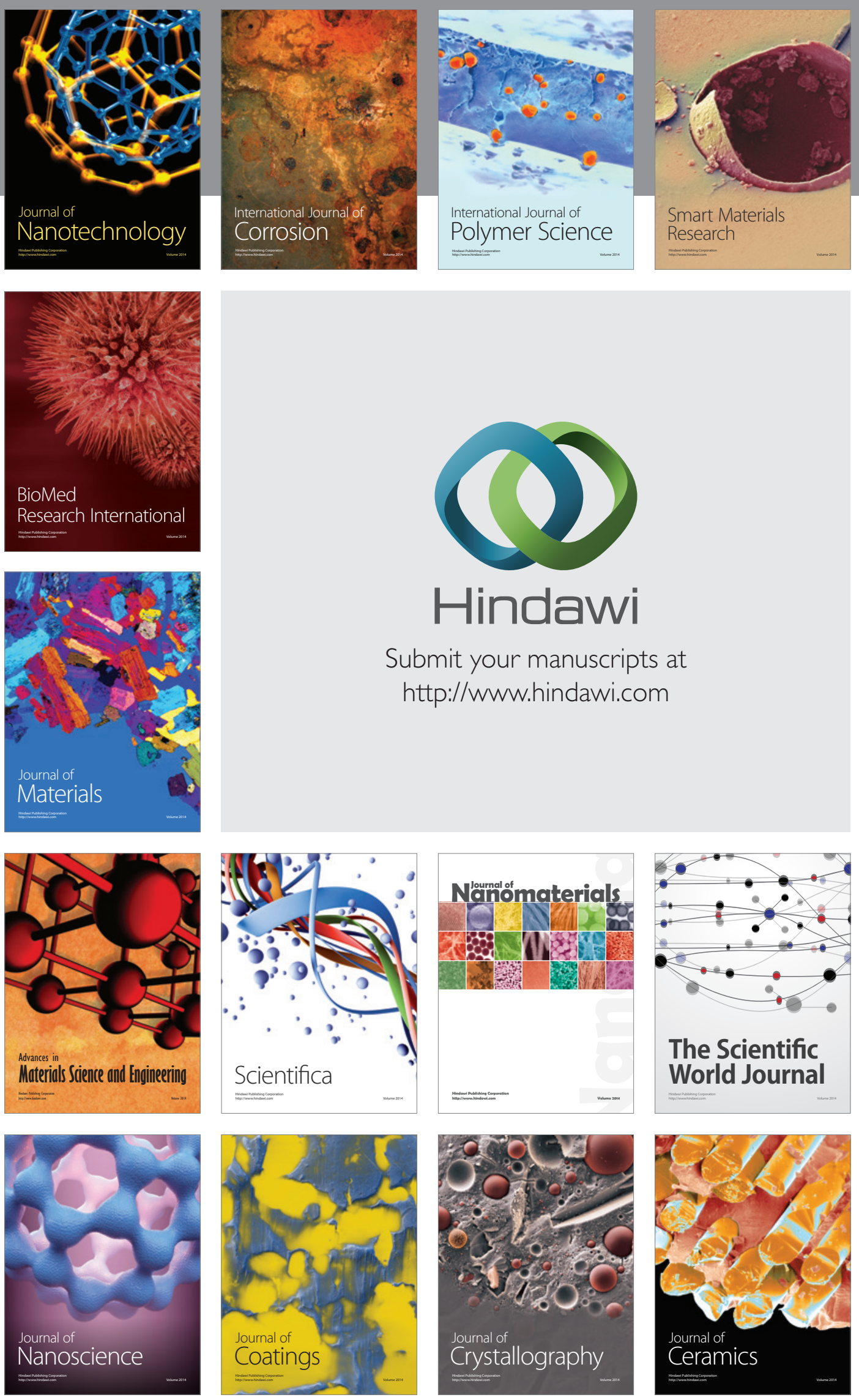

The Scientific World Journal

Submit your manuscripts at

http://www.hindawi.com

\section{World Journal}

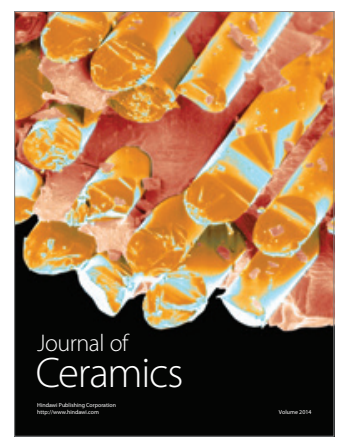

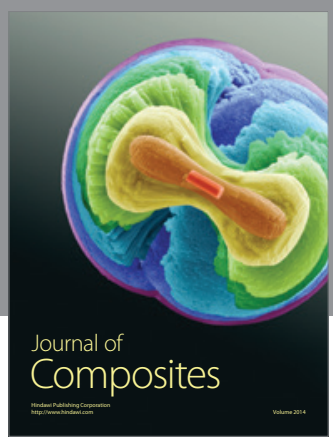
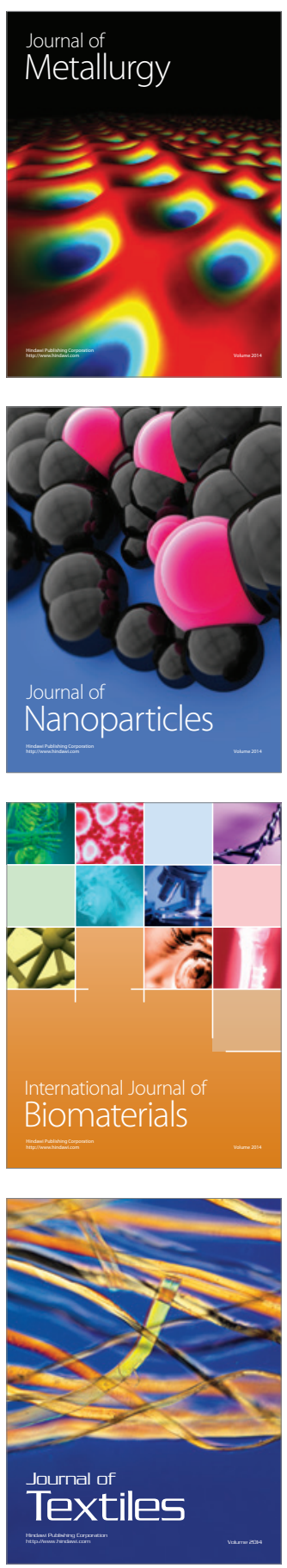